\title{
Brain natriuretic peptide is an indicator for early risk-stratification and management for COVID-19 patients
}

\author{
pengping $\mathrm{li}^{1}$, Wei $\mathrm{Wu}^{1}$, Tingting $\mathrm{Zhang}^{1}$, Ziyu Wang ${ }^{1}$, Ziyu Wang ${ }^{1}$, Mengyan Zhu ${ }^{1}$, Yuan \\ Liang $^{1}$, Wenhua You ${ }^{1}$, Kening Li $^{1}$, Rong Ding ${ }^{1}$, Bin Huang ${ }^{1}$, Lingxiang $\mathrm{Wu}^{1}$, Weiwei \\ Duan $^{1}$, Yi Han ${ }^{1}$, Xuesong $\mathrm{Li}^{1}$, Xin Tang ${ }^{1}$, Xin Wang ${ }^{2}$, Qianghu Wang ${ }^{1}$, Xinyi Xia ${ }^{3}$, \\ Hongshan Chen ${ }^{1}$, and Yong $\mathrm{Ji}^{1}$ \\ ${ }^{1}$ Nanjing Medical University \\ ${ }^{2}$ The University of Manchester \\ ${ }^{3}$ Nanjing University
}

October 1, 2020

\begin{abstract}
Background and Purpose: Coronavirus disease (COVID-19) has resulted in high mortality worldwide. However, information regarding cardiac markers for precise risk-stratification is limited. We aimed to discover a sensitive and reliable early-warning biomarker for optimizing management and improving COVID-19 patients' prognosis. Experiments Approach: This retrospectively single-center case series was conducted between February 4 and April 10, 2020. 3,046 consecutive COVID-19 patients who were receiving treatment at Wuhan Huoshenshan Hospital in China were included. Serum levels of cardiac markers and coronary artery disease (CAD) diagnosis were collected after admission. Single-cell RNA-sequencing was performed to analyze severe acute respiratory syndrome coronavirus 2 (SARS-CoV-2) receptor expression. Key Results: Median patient age was 60 years; $1,461(49.5 \%)$ were female, and 1,515 (51.3\%) were in a severe/critical condition. Compared to mild/moderate patients, severe/critical patients showed significantly higher levels of cardiac markers within the first week after admission. Among severe/critical COVID-19 patients, those with abnormal serum levels of brain natriuretic peptide had a significantly higher mortality than patients with normal levels. Severe/critical COVID-19 patients with pre-existing CAD (165/1,515) had more cases of abnormal brain natriuretic peptide levels than those without CAD. Enhanced SARS-CoV-2 receptor expression was observed in patients with CAD. Regression analysis revealed patients with elevated brain natriuretic peptide were at a higher risk of death. Conclusion and Implications: Brain natriuretic peptide is an effective biomarker for early risk assessment in COVID-19 patients with or without pre-existing CAD. Monitoring BNP status will improve the risk-stratification management and prognosis of patients within one week after admission.
\end{abstract}

\section{What is already known}

- COVID-19 patients develop myocardial injury during hospitalization face greater risk and have worse in-hospital outcomes

- However, information regarding cardiac markers for precise risk-stratification is limited

\section{What this study adds}

- BNP were higher in non-survivors on the first day and within the first week after admission

- BNP was an independent risk factor for COVID-19 patients in-hospital mortality

\section{Clinical significance}

$\mathrm{BNP}$ is an effective and reliable indicator for early risk-stratification and management for COVID-19 patients 


\section{Methods}

\subsection{Study Design and Participants}

This single-center retrospective study included consecutive patients diagnosed with COVID-19 at Wuhan Huoshenshan Hospital in China, between February 4 and April 10, 2020. The Wuhan Huoshenshan Hospital was built in ten days due to the extent of the pandemic, which exceeded the existing health system capacity. Most of the patients admitted to the Huoshenshan Hospital were transferred from other hospitals. The study design was approved by the institutional ethics board. Written informed consent was waived due to the urgency of the COVID-19 pandemic.

A total of 3,046 adult patients with COVID-19 were initially screened for the study. Individuals without information regarding disease severity, survival status, and patients with suspected CAD but not diagnosed were excluded. In the remaining cohort of 2,954 patients, 1,439 patients were mild/moderate cases and 1,515 were severe/critical cases. The disease severity was determined according to the clinical classification criterion in the Diagnosis and Treatment Protocol for Novel Coronavirus Pneumonia released by the National Health Commission of China (7th edition) (Pan et al., 2020). Patients who met any of the following criteria were diagnosed as a severe case: (1) shortness of breath defined by respiration rate [?]30 breaths/min, (2) oxygen saturation [?] 93 at rest, and (3) alveolar oxygen partial pressure/fraction of inspiration $\mathrm{O}_{2}\left(\mathrm{PaO}_{2} / \mathrm{FiO}_{2}\right)$ [?] $300 \mathrm{mmHg}(1 \mathrm{mmHg}=0.133 \mathrm{kPa})$. Patients whose pulmonary imaging showed significant progression of lesions $>50 \%$ within 24-48 hours were also treated as severe cases. Patients who met any of the following conditions were diagnosed as critically severe: (1) respiratory failure requiring mechanical ventilation, (2) shock, and (3) organ failure needing intensive care unit (ICU) monitoring and treatment. In addition, the severe/critical cohort was categorized into two groups based on the presence or absence of pre-existing CAD (165/1,515 [10.9\%] and 1350/1,515 [89.1\%], respectively), according to clinical diagnosis and/or medical history on admission.

\subsection{Data Collection}

Clinical information was collected during hospitalization by attending physicians. The radiologic results including chest radiography or computed tomography (CT) were retrieved from the radiology information system documents. Patient data, including demographics, medical history, comorbidities, laboratory examinations, viral load, SARS-CoV-2-specific IgG and IgM antibodies, and outcomes were collected from electronic medical records and analyzed.

\subsection{Cardiac biomarkers and heart damage}

Cardiac biomarkers are substances released into the blood when the heart is damaged or stressed and can be useful in the early prediction or diagnosis of disease (Halushka, Goodwin \& Halushka, 2019). Five cardiac biomarkers, namely, BNP, hs-TNI, $\alpha-\mathrm{HBDH}$, CK-MB, and LDH, were collected to evaluate heart function. Not all of the cardiac markers were tested for each patient during hospitalization. Therefore, patients were categorized according to the normal or abnormal results of the specific cardiac biomarker for each patient. Abnormal cardiac biomarker results were defined as serum levels above the upper reference limit.

\subsection{Transcriptional profiles}

To characterize the expression patterns of SARS-CoV-2 receptors in the heart, single-cell RNA sequencing (scRNA-seq) of 9,791 cells isolated from 12 normal human hearts was performing. The scRNA-seq data were obtained from the Gene Expression Omnibus (GEO) database with the accession number GSE109816 (Wang et al., 2020b) and processed using theSeurat toolkit (Butler, Hoffman, Smibert, Papalexi \& Satija, 2018). The expression profile of peripheral blood from 93 CAD patients and 48 healthy individuals was analyzed using the Agilent-067406 Human Microarray V4.0 platform (Aglient, Santa Clara, USA), and the data were downloaded from the GEO database with the accession number GSE113079 (Li et al., 2018).

\subsection{Statistical Analysis}


Continuous and categorical variables are presented as median (interquartile range $[\mathrm{IQR}]$ ) and number (\%), respectively. Comparisons between groups were performed using the Mann-Whitney U test, $\chi^{2}$ test, or Fisher's exact test where appropriate. Pearson's correlations were employed to study the association between laboratory parameters. All statistical analyses were conducted using R software. Multivariate Cox regressions, log-rank tests, and Kaplan-Meier curves to plot the cumulative rates of death were implemented using theSurvival package in $\mathrm{R}$ software. The receiver operating characteristic (ROC) curve to assess the overall accuracy of a prognostic marker was applied by using the R package ROCR. AP -value $<0.05$ was used to indicate statistical significance.

\section{RESULTS}

\subsection{Baseline Clinical Characteristics}

Of the initial 3,046 patients with COVID-19 enrolled in our study, 48 patients with no record of survival status, 29 patients without classification of disease severity, and 15 patients with suspected CAD but with no diagnosis were excluded. Among the final cohort of 2,954 patients, the median age was 60 years (range, 50-68 years), 1,461 (49.5\%) were female, and 1,515 (51.3\%) were severe/critical cases. The median hospital stay for severe/critical patients was significantly longer than that for mild/moderate cases. Compared with mild/moderate cases, severe/critical patients were more likely to experience chest congestion. Comorbidities were more prevalent among severe/critical patients compared to mild/moderate cases, including hypertension, diabetes, cardiovascular disease, cerebrovascular disease, cancer, and chronic obstructive pulmonary disease ( Table 1 ).

In terms of radiological and laboratory findings, severe/critical patients had more incidences of fuzzy boundaries and consolidation (Table 1) and significantly higher levels of C-reactive protein (CRP), D-dimer, interleukin-6 (IL-6), procalcitonin (PCT), and higher percentages of neutrophils (NEUT\%), lymphocytes (LYM\%), and monocytes (MONO\%) within the first week of admission (Figure 1) . Serum cardiac markers, namely, BNP, hs-TNI, $\alpha$-HBDH, CK-MB, and LDH, were also drastically elevated in severe/critical patients during the first week (Figure 1 ). In general, the results showed more pronounced activation of pathophysiological pathways in more severe cases of COVID-19.

\subsection{Cardiac Markers and Clinical Outcomes}

To evaluate the relationship between the degree of cardiac abnormality and disease outcome in patients with COVID-19, serum cardiac markers were measured. Due to the $0 \%$ mortality and favorable prognosis of mild/moderate patients, we focused on the 1,515 patients with severe/critical COVID-19 in the follow-up period.

Of the 1,515 severe/critical cases, BNP, hs-TNI, $\alpha$-HBDH, CK-MB, and LDH were detected in 835 patients, 660 patients, 1443 patients, 1442 patients, and 1443 patients, respectively. In total, 171 (20.5\%), 79 (12.0\%), $529(36.7 \%), 124(8.6 \%)$, and $447(3.1 \%)$ patients showed abnormal serum levels of BNP, hs-TNI, $\alpha$-HBDH, CK-MB, and LDH, respectively (Figure 2a ). Patients with an elevated level of a cardiac marker showed a significantly higher mortality than those with normal serum levels (Figure 2b ). The same trend was observed in the ICU admission rate (Figure 2c ). Figure 2d shows that the serum levels of BNP, hsTNI, $\alpha$-HBDH, CK-MB, and LDH were significantly higher during hospitalization in non-survivors than in survivors.

The scRNA-seq data of normal human heart tissue were analyzed and five cell types, namely, cardiomyocyte (CM), endothelial (EC), fibroblast (FB), macrophage (MP), and smooth muscle (SMC) cells, were identified (Figure 3a ). SARS-CoV-2 receptors ACE2, ANPEP, DPP4, and ENPEP were enriched in specific cell populations. ACE2 was mainly expressed in CM, EC, and FB cell types. ANPEP was enriched in CM, DPP4 was mainly expressed by CM and EC, and ENPEP was primarily expressed in CM and SMC (Figure $3 \mathrm{~b}$ and Figure 3c ).

\subsection{Heart Damage and Mortality Rate}


The 1,515 severe/critical patients were further categorized into groups according to the presence or absence of pre-existing CAD ( $n=165$ and $n=1,350$, respectively). Patients with pre-existing CAD had a significantly higher percent of elevated BNP than patients without CAD (Figure 4a ). To explore the underlying pathophysiological mechanism for the elevated levels of cardiac markers in COVID-19 patients with preexisting CAD, RNA-seq data from 93 patients with CAD and 48 healthy people were analyzed and compared. The results showed that compared with healthy controls, SARS-CoV-2 receptors, including Transmembrane Serine Protease 2 (TMPRSS2) and Glutamyl Aminopeptidase (ENPEP), were significantly upregulated in CAD (Figure 4b).

Compared to patients with normal levels of cardiac markers, those with abnormal levels of BNP, hs-TNI, $\alpha-\mathrm{HBDH}, \mathrm{CK}-\mathrm{MB}$, and LDH exhibited significantly higher mortality in both CAD and non-CAD groups (Figure 4c ). The same trend was observed for the ICU admission rate (Figure S1 ). The serum markers were then compared between non-survivors and survivors. The results showed that BNP, $\alpha$-HBDH, CK-MB, and LDH were significantly higher in non-survivors than in survivors for patients with pre-existing CAD; however, the significant difference was not observed for hs-TNI (Figure 4d) . In patients without preexisting CAD, all markers were significantly higher in non-survivors than in survivors during hospitalization (Figure 4e).

\subsection{BNP as a Risk-Stratification Biomarker}

The five cardiac markers were measured on the first day of admission. Notably, the median value of BNP was significantly higher in non-survivors than in survivors for those with pre-existing CAD $(911.3 \mathrm{pg} / \mathrm{mL}$ vs. $57.9 \mathrm{pg} / \mathrm{mL})$ and those without $(121 \mathrm{pg} / \mathrm{mL}$ vs. $0.01 \mathrm{pg} / \mathrm{mL})$. The median levels of hs-TNI, $\alpha-\mathrm{HBDH}$, and LDH were significantly higher in non-survivors than in survivors in patients without pre-existing CAD; however, a significant difference was not observed in patients with pre-existing CAD (Figure S2 ).

In non-survivors with pre-existing CAD, the median levels of BNP and hs-TNI within the first week showed a higher fold change (BNP: 5.8; hs-TNI: 7.5; $\alpha$-HBDH: 1.9; CK-MB: 0.81; LDH: 1.7) from the upper reference limit of each marker (Figure 5a ). In non-survivors without pre-existing CAD, the fold change for BNP, hsTNI, $\alpha$-HBDH:1.9, CK-MB, LDH was 1.4, 1.7, 2, 0.78, and 1.9, respectively (Figure 5b ). The serum level differences for the five markers within a week after admission in patients with or without pre-existing CAD showed that BNP, $\alpha-\mathrm{HBDH}$, and LDH values were significantly higher in non-survivors than in survivors regardless of pre-existing CAD. Levels of hs-TNI were significantly higher only in non-survivors than in survivors for patients without pre-existing CAD. Although CK-MB and BNP levels were significantly different between non-survivors and survivors, most data for non-survivors were within normal levels (Figure 5c and Figure 5d ). The change of BNP levels was the same as those mentioned above during hospitalization (Figure S3 ).

\subsection{Elevated BNP and Increased Mortality}

The 835 severe/critical patients with BNP values recorded during hospitalization were divided into BNP high $(\mathrm{n}=171)$ and BNP normal $(\mathrm{n}=664)$ groups based on the upper reference limit $(100 \mathrm{pg} / \mathrm{mL})$. Survival analysis was conducted on two groups using applying a mixed-effect Cox model adjusted for age, sex, comorbidities, and hs-TNI, $\alpha-\mathrm{HBDH}, \mathrm{CK}-\mathrm{MB}$, and LDH. As a result, the BNP normal group demonstrated a significantly lower risk of mortality (Figure 6a ). The BNP level correctly classified survivors and non-survivors with an area under the curve (AUC) of 0.81 with the ideal threshold value based on the ROC curve of 62.17 , a sensitivity of $71.7 \%$, and a specificity of $80.6 \%$ (Figure $\mathbf{6 b}$ ).

Pearson's correlations were used to study the association between BNP and other laboratory parameters. CRP (Figure 6c ), IL-6 (Figure 6d ), and D-dimer (Figure 6e ) showed a highly positive correlation with BNP. By contrast, BNP level was significantly negatively correlated with LYM\% (Figure 6f ).

\section{DISSCUSSION}

Our study provides detailed information about the association between cardiac markers and clinical outcomes of COVID-19 patients with or without pre-existing CAD. Patients with abnormal serum levels of cardiac 
markers, namely, BNP, hs-TNI, $\alpha-\mathrm{HBDH}$, CK-MB, and LDH, had a significantly higher mortality rate than patients with normal serum marker levels. In COVID-19 non-survivors with or without pre-existing CAD, the BNP level measured within one week after admission was a sensitive and reliable indicator of heart damage, showing a 5.8-fold and 1.4-fold significant increase from the upper reference limit, respectively. Moreover, our results demonstrated that BNP is an independent risk factor for in-hospital mortality. Additionally, the present study indicates that the BNP level is not only correlated with cardiac damage but also with the clinical parameters of other organ systems.

SARS-CoV-2 uses the ACE2 receptor to facilitate viral entry into target cells, causing multiorgan dysfunction (Zou, Chen, Zou, Han, Hao \& Han, 2020). It has been reported that the mechanism of acute myocardial injury caused by SARS-CoV-2 infection might be related to ACE2 (South, Diz \& Chappell, 2020). The scRNA-seq analysis in the present study showed that ACE2 was mainly expressed in CM, EC, and FB cell types, suggesting that SARS-CoV-2 receptor-related signaling pathways in myocardial injury may be mainly related to these cell populations.

Hoffman et al. (Zhang, Penninger, Li, Zhong \& Slutsky, 2020) recently demonstrated that the initial spike in protein priming by TMPRSS2 is essential for the entry and viral spread of SARS-CoV-2 through interaction with the ACE2 receptor (Stopsack, Mucci, Antonarakis, Nelson \& Kantoff, 2020). Our results showed that there was a significantly high expression of TMPRSS2 in CAD patients compared with healthy controls. This implies that upregulated TMPRSS2 may be the reason that patients with CAD represented a higher percentage of individuals with abnormal levels of cardiac markers after SARS-CoV-2 infection. The serine protease inhibitor camostat mesylate, approved in Japan to treat diseases, has been shown to block TMPRSS2 activity and is thus an interesting candidate to treat COVID-19 patients with pre-existing CAD (Kawase, Shirato, van der Hoek, Taguchi \& Matsuyama, 2012; Zhou et al., 2015).

Previous studies have investigated the association between myocardial injury markers and prognosis (Dong, Li, Yu, Lv \& Chen, 2020; Lippi, Lavie \& Sanchis-Gomar, 2020). However, evidence on markers that are appropriate for monitoring heart damage in a timely manner is lacking. Because of limited space in ICUs, the triage rules for access to intensive care are becoming tougher and tougher. In this study, an extensive investigation of various clinical indices revealed that BNP is a sensitive and reliable biomarker for prognosis in COVID-19 patients. Particularly, compared with survivors, the median value of BNP was strikingly higher in non-survivors with or without CAD. Remarkably, abnormal elevation of serum BNP can accurately predict the risk of mortality on the day of admission through the first week after admission, which provides an early and wide window for clinical monitoring to strengthen cardio-protective treatment. $\alpha$-HBDH and LDH could also be used as indicators in risk stratification management; however, they are not specific indicators of heart damage and had a less drastic change in levels compared to BNP.

As a classic serum indicator for cardiac dysfunction, the detection of BNP is widely used in clinical practice and is, therefore, easy to implement in hospitals at all levels. Our results showed BNP is a powerful biomarker to predict the outcomes of COVID-19 patients and it should be detected on admission to guide therapy. Monitoring serum BNP status will improve the risk-stratified management and prognosis of COVID-19 patients with or without CAD on admission.

Some limitations exist in the present study. First, data from larger populations and multiple centers are needed to further verify the results. Second, some data regarding heart damage, such as echocardiography, magnetic resonance, and electrocardiography, were incomplete due to the limited conditions in the isolation ward. Third, causes of death may involve multiple organ dysfunctions in most cases, and it is difficult to differentiate the heart damage as the main and direct cause in an individual case.

In conclusion, COVID-19 patients with pre-existing CAD represented a higher abnormal percentage of cardiac markers, accompanied by high mortality and ICU admission rate. Of note, COVID-19 patients without preexisting CAD who represented abnormal levels of cardiac markers also had high mortality. BNP is a sensitive and reliable biomarker for early warning of high mortality risk at the time of admission and through the first week. Monitoring BNP status as early as possible and providing cardioprotective combination therapy will 
improve the risk-stratified management and prognosis of patients.

\section{Abbreviations}

COVID-19: Coronavirus disease; CAD: coronary artery disease; SARS-CoV-2: severe acute respiratory syndrome coronavirus 2; hs-TNI: high-sensitivity troponin I; $\alpha$-HBDH: $\alpha$-hydroxybutyrate dehydrogenase; CK-MB: creatine kinase-MB; LDH: lactate dehydrogenase; BNP: brain natriuretic peptide; CT: computed tomography; ICU: intensive care unit; scRNA-seq: single-cell RNA sequencing; GEO: Gene Expression Omnibus; ROC: receiver operating characteristic; CRP: C-reactive protein; IL-6: interleukin-6; PCT: procalcitonin; NEUT\%: percentages of neutrophils; LYM\%: percentages of lymphocytes; MONO\%: percentages of monocytes.

\section{References}

Bodor GS (2016). Biochemical Markers of Myocardial Damage. EJIFCC 27: 95-111.

Butler A, Hoffman P, Smibert P, Papalexi E, \& Satija R (2018). Integrating single-cell transcriptomic data across different conditions, technologies, and species. Nat Biotechnol 36: 411-420.

Dong N, Cai J, Zhou Y, Liu J, \& Li F (2020). End-stage Heart Failure with COVID-19: Strong Evidence of Myocardial Injury by 2019-nCoV. JACC Heart Fail.

Dong Y, Li X, Yu Y, Lv F, \& Chen Y (2020). JAK/STAT signaling is involved in IL-35-induced inhibition of hepatitis B virus antigen-specific cytotoxic T cell exhaustion in chronic hepatitis B. Life Sci 252: 117663.

Doust JA, Pietrzak E, Dobson A, \& Glasziou PP (2005). How well does B-type natriuretic peptide predict death and cardiac events in patients with heart failure: systematic review. Brit Med J 330: 625-627.

Fu Y, Cheng Y, \& Wu Y (2020). Understanding SARS-CoV-2-Mediated Inflammatory Responses: From Mechanisms to Potential Therapeutic Tools. Virol Sin.

Guan WJ, Ni ZY, Hu Y, Liang WH, Ou CQ, He JX, et al. (2020). Clinical Characteristics of Coronavirus Disease 2019 in China. N Engl J Med 382: 1708-1720.

Guo T, Fan Y, Chen M, Wu X, Zhang L, He T, et al. (2020). Cardiovascular Implications of Fatal Outcomes of Patients With Coronavirus Disease 2019 (COVID-19). JAMA Cardiol.

Halushka PV, Goodwin AJ, \& Halushka MK (2019). Opportunities for microRNAs in the Crowded Field of Cardiovascular Biomarkers. Annu Rev Pathol 14: 211-238.

Huang C, Wang Y, Li X, Ren L, Zhao J, Hu Y, et al. (2020). Clinical features of patients infected with 2019 novel coronavirus in Wuhan, China. Lancet 395: 497-506.

Kawase M, Shirato K, van der Hoek L, Taguchi F, \& Matsuyama S (2012). Simultaneous treatment of human bronchial epithelial cells with serine and cysteine protease inhibitors prevents severe acute respiratory syndrome coronavirus entry. J Virol 86: 6537-6545.

Lee YJ, Lee J, Park YS, Lee SM, Yim JJ, Yoo CG, et al. (2013). Predictors of cardiogenic and noncardiogenic causes in cases with bilateral chest infiltrates. Tuberc Respir Dis (Seoul) 74:15-22.

Li L, Wang L, Li H, Han X, Chen S, Yang B, et al. (2018). Characterization of LncRNA expression profile and identification of novel LncRNA biomarkers to diagnose coronary artery disease. Atherosclerosis 275: 359-367.

Lippi G, Lavie CJ, \& Sanchis-Gomar F (2020). Cardiac troponin I in patients with coronavirus disease 2019 (COVID-19): Evidence from a meta-analysis. Prog Cardiovasc Dis.

Pan Y, Guan H, Zhou S, Wang Y, Li Q, Zhu T, et al. (2020). Initial CT findings and temporal changes in patients with the novel coronavirus pneumonia (2019-nCoV): a study of 63 patients in Wuhan, China. Eur Radiol 30: 3306-3309. 
Shi S, Qin M, Shen B, Cai Y, Liu T, Yang F, et al. (2020). Association of Cardiac Injury With Mortality in Hospitalized Patients With COVID-19 in Wuhan, China. JAMA Cardiol.

Singhal T (2020). A Review of Coronavirus Disease-2019 (COVID-19). Indian J Pediatr 87: 281-286.

South AM, Diz DI, \& Chappell MC (2020). COVID-19, ACE2, and the cardiovascular consequences. Am J Physiol Heart Circ Physiol 318: H1084-H1090.

Stopsack KH, Mucci LA, Antonarakis ES, Nelson PS, \& Kantoff PW (2020). TMPRSS2 and COVID-19: Serendipity or Opportunity for Intervention? Cancer Discov.

Wang D, Hu B, Hu C, Zhu F, Liu X, Zhang J, et al. (2020a). Clinical Characteristics of 138 Hospitalized Patients With 2019 Novel Coronavirus-Infected Pneumonia in Wuhan, China. JAMA.

Wang L, Yu P, Zhou B, Song J, Li Z, Zhang M, et al. (2020b). Single-cell reconstruction of the adult human heart during heart failure and recovery reveals the cellular landscape underlying cardiac function. Nat Cell Biol 22: 108-119.

Zhang H, Penninger JM, Li Y, Zhong N, \& Slutsky AS (2020). Angiotensin-converting enzyme 2 (ACE2) as a SARS-CoV-2 receptor: molecular mechanisms and potential therapeutic target. Intensive Care Med 46: 586-590.

Zheng Z, Peng F, Xu B, Zhao J, Liu H, Peng J, et al. (2020). Risk factors of critical \& mortal COVID-19 cases: A systematic literature review and meta-analysis. The Journal of infection.

Zhou Y, Vedantham P, Lu K, Agudelo J, Carrion R, Jr., Nunneley JW, et al. (2015). Protease inhibitors targeting coronavirus and filovirus entry. Antiviral Res 116: 76-84.

Zou X, Chen K, Zou J, Han P, Hao J, \& Han Z (2020). Single-cell RNA-seq data analysis on the receptor ACE2 expression reveals the potential risk of different human organs vulnerable to 2019-nCoV infection. Front Med.

Tables

Table 1. Demographics and Clinical Characteristics of Patients with COVID-19

\section{Figure Legends}

Figure 1. Serum cardiac markers drastically elevated in severe/critical patients during the first week. Levels of 12 multiple organ dysfunction indicators in mild/moderate (M) and severe/critical (S) groups during the first week of hospitalization. The difference of each indicator between the two groups is significant (two-sided Wilcoxon test, $P<0.001$ ).

Figure 2. Severe/critical COVID-19 patients with evidence of cardiac damage have poor clinical outcomes. (A) . Number of patients with abnormal and normal levels of BNP, hs-TNI, $\alpha$-HBDH, CK-MB, and LDH. The mortality (B) and ICU admission rate (C) of severe/critical COVID-19 patients with abnormal serum levels of BNP, hs-TNI, $\alpha-\mathrm{HBDH}$, CK-MB, and LDH. (D) Serum levels of BNP, hsTNI, $\alpha-\mathrm{HBDH}, \mathrm{CK}-\mathrm{MB}$, and LDH during hospitalization for non-survivors and survivors. Shaded regions represent a $95 \%$ confidence interval.

Figure 3. scRNA-seq analysis of SARS-CoV-2 receptors in heart specific cell populations. (A) The t-distributed Stochastic Neighbor Embedding (t-SNE) diagram shows the main cell types in healthy heart tissue. Each dot represents a cell, and each color represents a cell type. (B) Feature maps and (C)violin diagrams show SARS-CoV-2 receptors are enriched in specific cell populations in healthy heart tissues.

Figure 4. Severe/critical COVID-19 patients with abnormal cardiac markers exhibited higher mortality. (A)Percent of abnormal cardiac markers in patients with and without pre-existing CAD. (B) 
Expression of SARS-CoV-2 receptors, TMPRSS2 and ENPEP, in coronary artery disease and healthy controls.(C) The mortality rate of COVID-19 patients with cardiac markers abnormality. (D-E) Median levels of cardiac markers in survivors and non-survivors during hospitalization. $* * * P<0.001$; NS: not significant.

Figure 5. BNP is a Risk-Stratification Biomarker for COVID-19 patients. (A-B) Fold change of cardiac markers relative to the upper reference within one week after admission. (C-D) The fluctuation of serum levels of cardiac markers within one week after admission.

CAD: COVID-19 patients with pre-existing CAD; non-CAD: COVID-19 patients without pre-existing CAD.

Figure 6. Abnormal BNP level is correlated with increased mortality of COVID-19 patients. (A) Mortality during hospitalization between patients with elevated BNP vs. normal BNP levels. Log-rank test shows statistical significance. The table below shows the number of people still alive at different time points.(B) ROC curve of BNP to predict survivors and non-survivors.(C-F) Correlation between BNP and other laboratory indicators, including CRP (C), IL-6 (D) , D-dimer (E) , and LYM\% (F). The 95\% confidence interval represented by shaded regions.

\section{SUPPORTING INFORMATION}

\section{Supplemental Figures and Figure Legends}

Figure S1. The ICU admission rate of COVID-19 patients with cardiac markers abnormality

Figure S2. Median levels of the cardiac markers on the first day after admission for four groups of patients (non-survivors with CAD, survivors with CAD, non-survivors without CAD, survivors without CAD). The dotted line shows the upper reference limit of the corresponding marker.

Figure S3 . The fluctuation of serum levels for 5 cardiac markers in survivors and non-survivors with and without CAD during hospitalization. The $\mathrm{x}$-axis represents the admission time. The red dotted line represents the reference limit of the marker. (Number): the fold change for median level versus the reference limit value for each marker.

\section{Hosted file}

Table 1.pdf available at https://authorea.com/users/362745/articles/483807-brain-natriureticpeptide-is-an-indicator-for-early-risk-stratification-and-management-for-covid-19patients
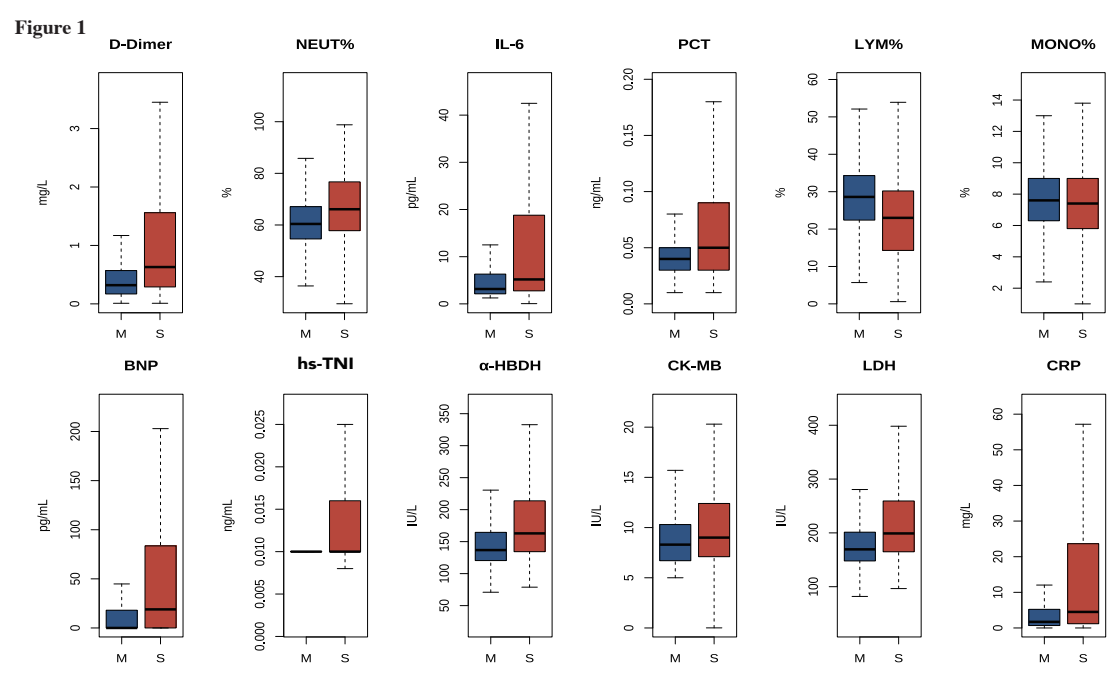
Figure 2

(a)

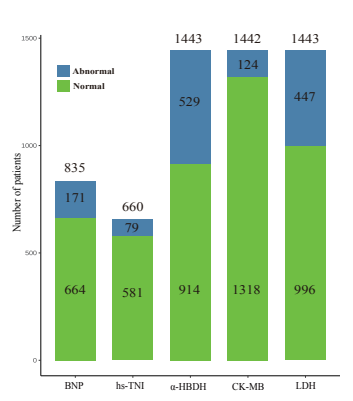

(d)
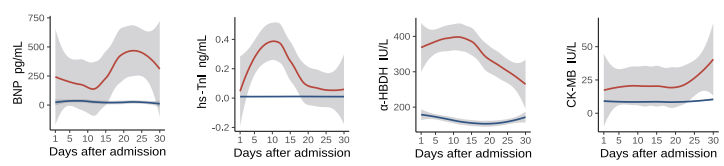

二
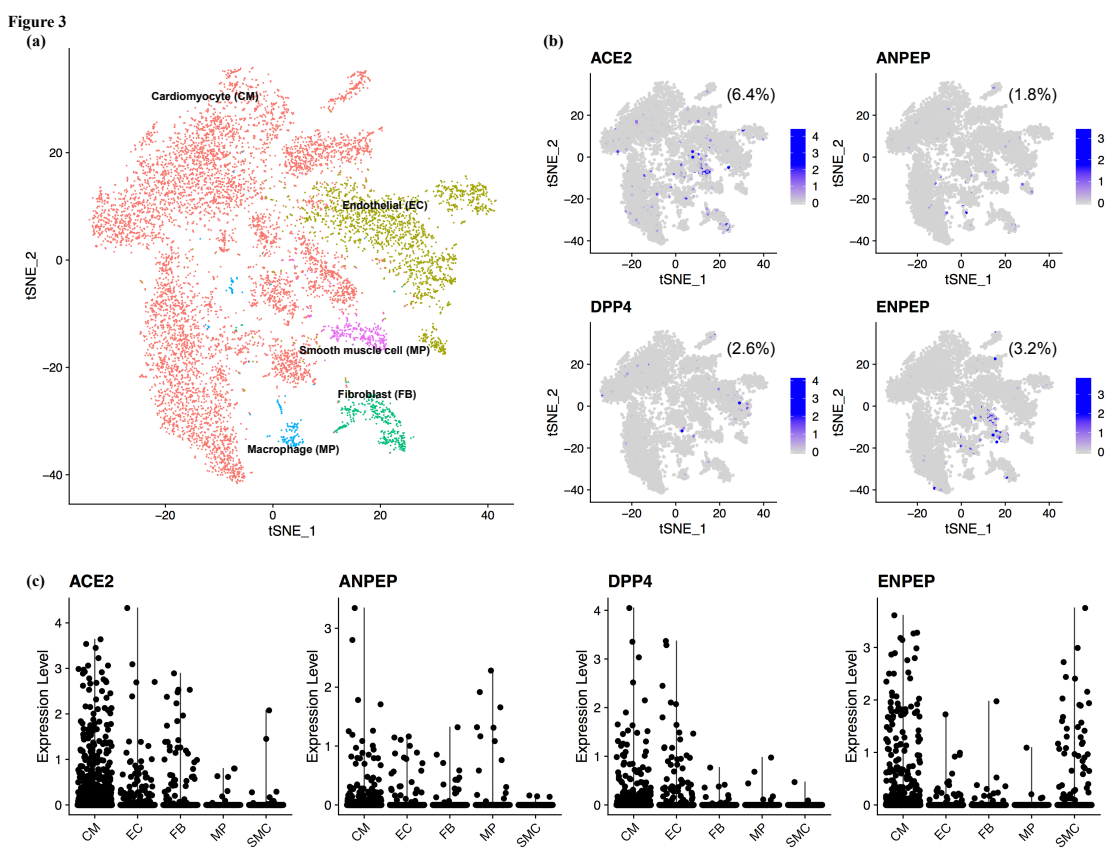
Figure 4

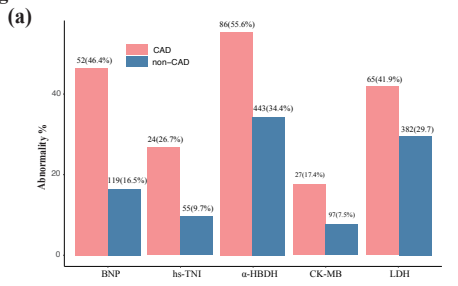

(c)

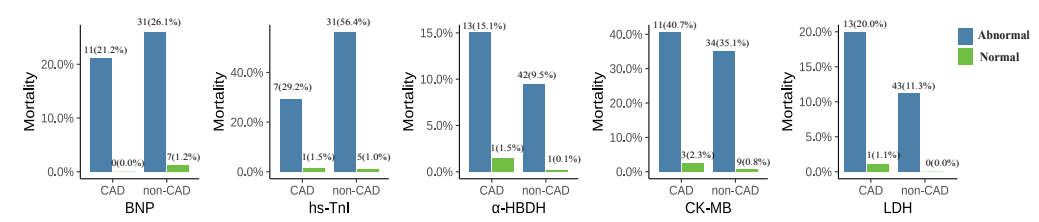

(d)
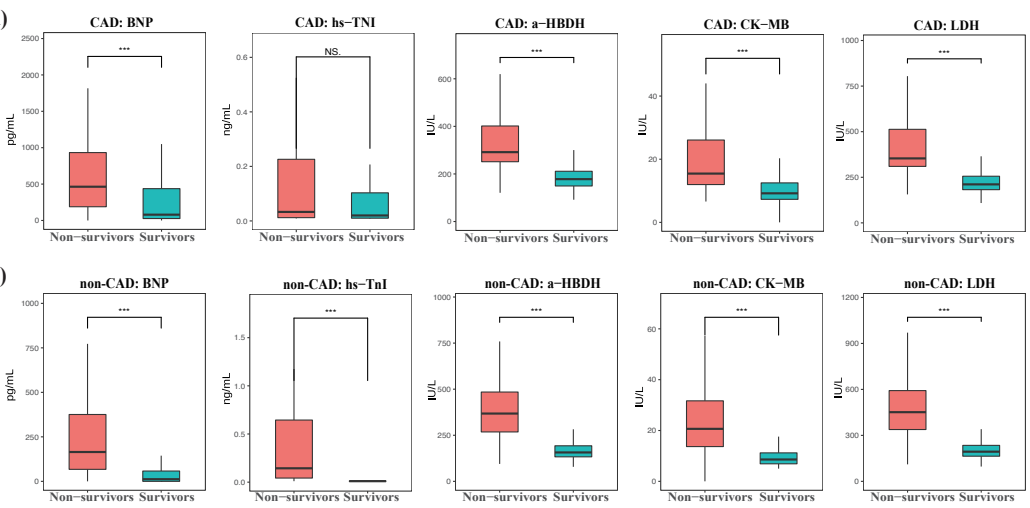
Figure 5

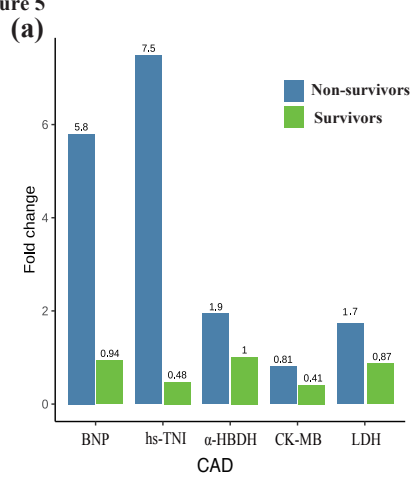

(b)

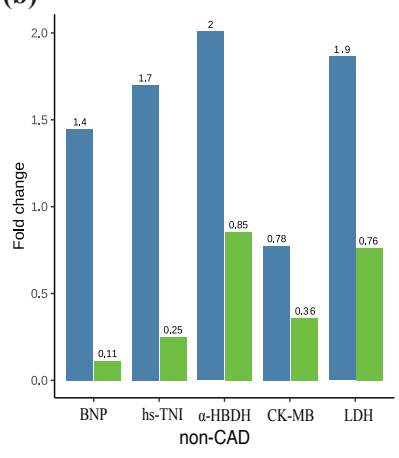

(c)
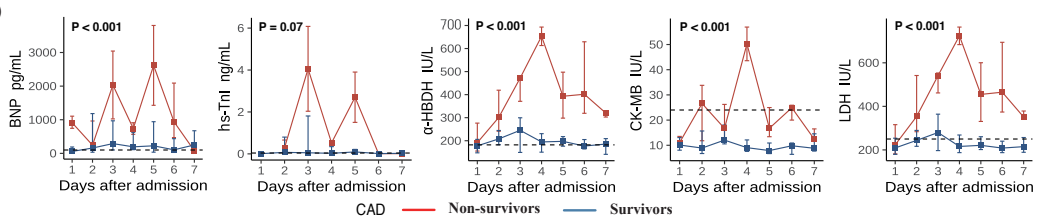

(d)

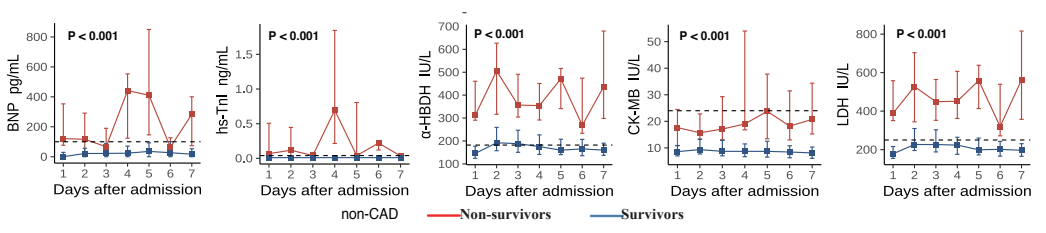

Figure 6

(a)

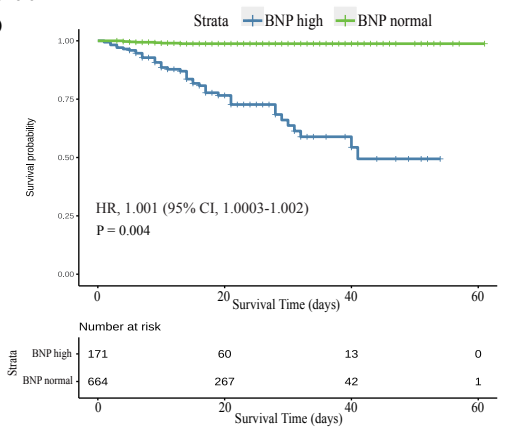

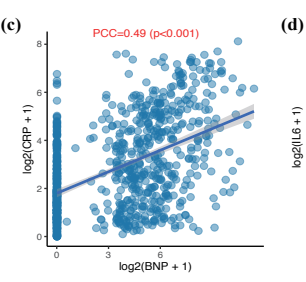

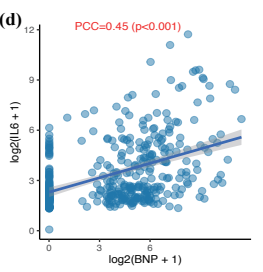

(b)
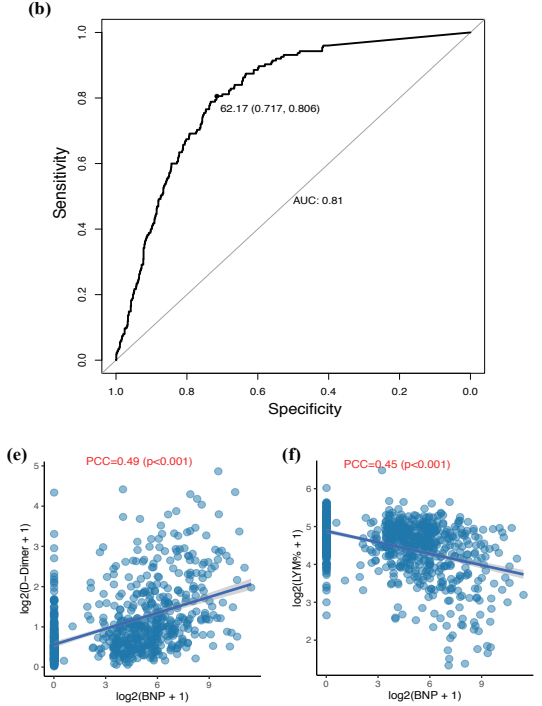